\title{
Detecção de maculopatia hipotônica subclínica pelo OCT III após cirurgia filtrante
}

\author{
Detection of subclinical hypotony maculopathy with OCT III after filtration surgery
}

\author{
Mônica Weyll ${ }^{1}$ \\ Adriana Gilio ${ }^{2}$ \\ Aline Barbosa ${ }^{3}$ \\ Aguida Aparecida Nicoli ${ }^{4}$ \\ Regina Cele Silveira ${ }^{5}$
}

\begin{tabular}{|c|}
\hline RESUMO \\
\hline Objetivo: Detectar possíveis sinais de maculopatia hipotônica subclínica \\
pormeio da OCT III em pacientes submetidos à cirurgia filtrante. Métodos: \\
Pacientes que realizaram procedimento cirúrgico filtrante com pressão \\
intra-ocular menor que $9 \mathrm{mmHg}$ submeteram-se ao exame OCT III. \\
Resultados: Sete $(87,50 \%)$ pacientes apresentaram diagnóstico prévio \\
de glaucoma crônico simples e $1(12,50 \%)$ de glaucoma agudo de ângulo \\
fechado. Apenas duas pacientes $(25,00 \%)$ apresentaram maculopatia \\
hipotônica detectada pela OCT III. Conclusão: A OCT III parece ser um \\
bom método diagnóstico de maculopatia hipotônica subclínica em pa- \\
cientes submetidos à cirurgia filtrante convencional.
\end{tabular}

Descritores: Hipotensão ocular/etiologia; Cirurgia filtrante; Tomografia de coerência óptica; Trabeculectomia/efeitos adversos

\section{INTRODUÇÃO}

Maculopatia hipotônica é uma complicação pós-cirúrgica filtrante ${ }^{(1-4)}$. Sua causa ainda é desconhecida, mas sabe-se que a aplicação intra-operatória de mitomicina $\mathrm{C}$ é um fator predisponente ${ }^{(2)}$. Acredita-se que a mesma reduza o fluxo de humor aquoso, provavelmente pela destruição do corpo ciliar ${ }^{(2-3)}$.

Dois mecanismos podem contribuir para o aparecimento da hipotonia: aumento no fluxo de humor aquoso, como ocorre em casos de bolha hiperfiltrante e vazamento conjutival, ou redução em sua produção, geralmente decorrente por exemplo, de descolamento ciliocoroidal e de processo inflamatório $^{(1-5)}$.

A hipotonia com pressão intra-ocular menor que $5 \mathrm{mmHg}$ pode ser compatível com boa acuidade visual ${ }^{(1)}$. Porém, quando prolongada, pode causar dano macular permanente, redução da produção de humor aquoso, catarata, descolamento ciliocoroidal, falência de fístula, edema corneano e hemorragia coroidal ${ }^{(1-4)}$. Bolha hiperfiltrante também pode causar maculopatia hipotônica, principalmente se a mesma persistir por pelo menos três meses $^{(3)}$. Outros fatores são raça branca, jovens e míopes ${ }^{(3-6)}$. No exame de fundo de olho observa-se efusão coroidal e estrias retinianas ${ }^{(4,6)}$.

A OCT III permite, de forma não invasiva, obter-se imagens topográficas da retina em corte axial de $10 \mu^{(4,7-8)}$. É opticamente equivalente à ultrasonografia de alta resolução para avaliar camada de fibras nervosas ${ }^{(7-8)}$.

O estudo visa detectar possíveis sinais de maculopatia hipotônica subclínica através da OCT III em pacientes submetidos à cirurgia filtrante.

\section{MÉTODOS}

Este é um trabalho prospectivo do setor de glaucoma do Complexo 
Hospitalar Padre Bento de Guarulhos (CHPB) e Universidade Federal de São Paulo (UNIFESP).

Foram critérios de inclusão: aceitar o termo de consentimento, não ter realizado cirurgia oftalmológica prévia e ausência de complicações no intra-operatório.

Os critérios de exclusão do trabalho foram: complicação pós-operatória, pressão intra-ocular maior que $9 \mathrm{mmHg}$ desde o primeiro dia pós-operatório até o décimo quarto dia, recusa em consentir a participação no estudo e idade menor que 18 anos.

A trabeculectomia foi realizada com a técnica convencional, porém com mitomicina C diluída a $0,2 \mathrm{mg} / \mathrm{ml}$ com exposição de 2 min sob o Tenon e lavada copiosamente com $40 \mathrm{ml}$ de solução BSS no intra-operatório.

Após procedimento cirúrgico, os pacientes foram avaliados no $1^{\circ}, 7^{\circ}$ e $14^{\circ}$ pós-operatório, sendo realizado exame oftalmológico completo. Foram avaliados a acuidade visual com e sem correção, exame biomicroscópico e tonometria com o tonômetro de Goldmann. Todos os pacientes foram submetidos ao exame fundoscópico no $7^{\circ}$ dia pós-operatório, sendo considerados para realizarem OCT III apenas aqueles com PIO menor que $9 \mathrm{mmHg}$ e sem alterações compatíveis com maculopatia hipotônica ao exame com a lente de 90 D. Entre o $7^{\circ}$ e $14^{\circ}$ dia pós-operatório, os mesmos foram encaminhados para realização do OCT III no setor de glaucoma da UNIFESP-EPM, o qual foi confrontado com o exame oftalmológico.

\section{RESULTADOS}

Foram estudados oito olhos de pacientes submetidos à trabeculectomia com mitomicina C que realizaram OCT III (Tabela). Todos do sexo feminino com idade entre 35 e 75 anos.

Das oito pacientes estudadas, 7 (87,5\%) delas apresentaram diagnóstico prévio de glaucoma crônico simples e 1 $(12,5 \%)$ de glaucoma agudo de ângulo fechado.

Todos os olhos estudados não apresentavam história prévia de cirurgia filtrante e eram fácicos.

Nenhuma das pacientes evoluiu com piora da acuidade visual após o procedimento cirúrgico, nem apresentaram sinais fundoscópicos de maculopatia hipotônica.

Apenas duas pacientes $(25,00 \%)$ apresentaram maculo- patia hipotônica detectada pela OCT III, ambas apresentando pressão intra-ocular de $6 \mathrm{mmHg}$ e $4 \mathrm{mmHg}$, respectivamente, de acordo com a ordem na tabela.

\section{DISCUSSÃO}

Apesar do pequeno número de pacientes da amostragem, pode-se observar que foi freqüente a presença de maculopatia hipotônica subclínica, que ocorreu em 25,0\% dos casos. Karasheva et al. e Aydin et al. observaram um espessamento da região foveal, após cirurgia filtrante, com duração de até um mês, sem comprometimento da câmara anterior, sugerindo que a hipotonia pós-operatória está associada à técnica cirúrgica utilizada $^{(5,8)}$. No presente estudo, a técnica foi a mesma para todos os pacientes sumetidos ao procedimento cirúrgico.

Zacaria et al. detectou alta incidência de hipotonia após trabeculectomia com mitomicina C (20,0\% em olhos com exposição de 3,5 min e 35,7\% em períodos maiores), principalmente em pacientes sem história prévia de procedimento cirúrgico filtrante ou valvulado, o qual se desenvolveu em períodos de exposição mais elevado ao antimetabólito diluído a $0,4 \mathrm{mg} / \mathrm{ml}^{(1)}$. No estudo atual, observamos uma frequiência relativamente alta para o número de pacientes estudados, embora a diluição usada $(0,2 \mathrm{mg} / \mathrm{ml})$ e o tempo de exposição à mitomicina $\mathrm{C}(2,0 \mathrm{~min})$ tenham sido reduzidos, comparados ao estudo anterior.

Bashford et al. observaram que a duração da hipotonia pósoperatória não se correlacionou à piora da acuidade visual, o que foi notado no presente estudo, no qual nenhuma das pacientes apresentou baixa acuidade visual após a cirurgia ${ }^{(6)}$.

São necessários mais estudos para avaliar a importância do OCT III na detecção da maculopatia hipotônica subclínica, bem como para avaliar a necessidade de seu uso rotineiro no pós-operatório dos pacientes submetidos à cirurgia filtrante.

\section{CONCLUSÃO}

A OCT III parece ser um bom método no diagnóstico de maculopatia hipotônica subclínica em pacientes submetidos à cirurgia filtrante convencional.

\begin{tabular}{|c|c|c|c|c|c|c|}
\hline Paciente & Sexo & AV prévia & Classificação do glaucoma & Tipo de cirurgia & OCT III & AV posterior \\
\hline 1 & $\mathrm{~F}$ & $20 / 200$ & Crônico simples & TREC & Não & $20 / 30$ \\
\hline 2 & $\mathrm{~F}$ & $20 / 20$ & Crônico simples & TREC & $\operatorname{Sim}(\mathrm{MH})$ & $20 / 20$ \\
\hline 3 & $\mathrm{~F}$ & $20 / 40$ & Crônico simples & TREC & Difícil avaliar & $20 / 40$ \\
\hline 4 & $\mathrm{~F}$ & MM & Âgudo fechado & TREC & Difícil avaliar & MM \\
\hline 5 & $\mathrm{~F}$ & MM & Crônico simples & TREC & Difícil avaliar & $10 / 30$ \\
\hline 6 & $\mathrm{~F}$ & $20 / 200$ & Crônico simples & TREC & Não & $20 / 60$ \\
\hline 7 & $\mathrm{~F}$ & $10 / 200$ & Crônico simples & TREC & Não & $20 / 100$ \\
\hline 8 & $\mathrm{~F}$ & $20 / 100$ & Crônico simples & TREC & $\operatorname{Sim}(\mathrm{MH})$ & $20 / 40$ \\
\hline
\end{tabular}




\section{ABSTRACT}

Purpose: To detect nondiagnostic hypotony maculopathy by OCT III after filtration surgery. Methods: After surgery, patients with intraocular pressure less than $9 \mathrm{mmHg}$ were submitted to OCT III examination. Results: Seven $(87.50 \%)$ patients with previous diagnosis of open angle glaucoma and one $(12.50 \%)$ of them with acute angle closure glaucoma. Two patients $(25.00 \%)$ presented hypotony maculopathy on OCT III examination. Conclusion: OCT III examination seems to be a good diagnostic method to detect subclinical hypotony maculopathy after filtration surgery.

Keywords: Ocular hypotension/etiology; Filtering surgery; Tomography, optical coherence; Trabeculectomy/adverse effects

\section{REFERÊNCIAS}

1. Zacharia PT, Deppermann SR, Schuman JS. Ocular hypotony after trabeculectomy with mitomycin C. Am J Ophthalmol. 1993;116(3):314-26.

2. Nuyts RM, Greve EL, Geijssen HC, Langerhorst CT. Treatment of hypotonous maculopathy after trabeculectomy with mitomycin C. Am J Ophthalmol. 1994;118(3):322-31.

3. Costa VP, Wilson RP, Moster MR, Schmidt CM, Gandham S. Hypotony maculopathy following the use of topical mitomycin $\mathrm{C}$ in glaucoma filtration surgery. Ophthalmic Surg. 1993;24(6):389-94.

4. Azuara-Blanco A, Katz LJ. Dysfunctional filtering blebs. Surv Ophthalmol. 1998;43(2):93-126. Review.

5. Karasheva G, Goebel W, Klink T, Haigis W, Grehn F. Changes in macular thickness and depth of anterior chamber in patients after filtration surgery. Graefes Arch Clin Exp Ophthalmol. 2003;241(3):170-5.

6. Bashford KP, Shafranov G, Shields MB. Bleb revision for hypotony maculopathy after trabeculectomy. J Glaucoma. 2004;13(3):256-60.

7. Schuman JS, Hee MR, Puliafito CA, Wong C, Pedut-Kloizman T, Lin CP, et al. Quantification of nerve fiber layer thickness in normal and glaucomatous eyes using optical coherence tomography. Arch Ophthalmol. 1995;113(5):586-96.

8. Aydin A, Wollstein G, Price LL, Fujimoto JG, Schuman JS. Optical coherence tomography assessment of retinal nerve fiber layer thickness changes after glaucoma surgery. Ophthalmology. 2003;110(8):1506-11. 